\title{
Proteasome Inhibitor Carfilzomib Enhances the Anticancer Effect of Paclitaxel in MDA-MB-231 Breast Cancer Cells
}

\author{
HATICE TERZi ${ }^{*}$, E. MUSTAFA ${ }^{1}$, MERVE ERGÜL ${ }^{2}$, A. AHMET ${ }^{3}$ AND S. MEHMET \\ Medical Faculty, Department of Hematology, ${ }^{1}$ Department of Biochemistry, ${ }^{2}$ Faculty of Pharmacy, Department of \\ Pharmacology, Faculty of Pharmacy, ${ }^{3}$ Medical Faculty, Department of Pharmacology, Sivas Cumhuriyet University, Sivas, \\ Turkey
}

Terzi et al.: Carfilzomib Enhances the Anticancer Effect of Paclitaxel

This study was aimed to determine the anticancer efficacy of the combination of paclitaxel, the standard treatment of breast cancer, and carfilzomib, which is a proteasome inhibitor, in breast cancer cells in vitro. Paclitaxel, carfilzomib and their combinations at various concentrations were added to MDA-MB-231 human breast cancer cells, and cell viability was detected using the XTT assay. Combination index values were determined using the Chou-Talalay method. Apoptotic effect and cell cycle arrest of single administrations and combinations of these agents were also evaluated using the flow cytometry. According to the results of the XTT assay, the combination produced a greater anticancer effect than that produced by both agents administered alone. Chou-Talalay approaches exhibited that the combination of paclitaxel and carfilzomib demonstrated a synergistic effect. The flow analysis showed that the combinations have induced a cell cycle arrest of MDA-MB-231 cells at G2/M phase and significantly induced apoptosis. The present study revealed that carfilzomib could significantly increase the efficacy of paclitaxel in human breast cancer cells in vitro. It is recommended that carfilzomib to be used along with paclitaxel in metastatic breast cancer patients to increase the anticancer effect and reduce potential toxicity-related side effects.

Key words: Apoptosis, breast cancer, carfilzomib, cell cycle, paclitaxel 
Breast cancer is one of the most frequently diagnosed cancer types in women ${ }^{[1,2]}$. The incidence of breast cancer increases yearly due to genetic factors, lifestyle, and environmental factors and is tending towards younger women $^{[3,4]}$. Nowadays, breast cancer treatments, including surgery, adjuvant chemotherapy, radiotherapy, and immunotherapy are mainly comprehensive. Among them, chemotherapy is a meaningful way to mitigate advanced breast cancer pain and prolong the survival period. In this context, taxanes have been used in breast cancer therapy since the 1990s and have been displayed to be efficient against early breast cancer as well as metastatic breast cancer. Frequently used taxanes include the standard taxane paclitaxel (PTX) and docetaxel, which is the semi-synthetic derivative of PTX. These two drugs act as microtubule-stabilizing agents, causing cell death via microtubule stabilization and beta-tubulin binding that results in apoptosis due to $\mathrm{G} 2 / \mathrm{M}$ arrest ${ }^{[5]}$. PTX should be administered as a first-line, however, be scheduled with other drugs ${ }^{[6,7]}$. Resistance to PTX gradually emerges in many patients following the drug's widespread use.

Multi-drug resistance reduces the efficacy of chemotherapy ${ }^{[8]}$. Advanced-stage breast cancer is another critical issue that can metastasize to distant tissues of the body, as a result reducing the effectiveness of radiation therapy or chemotherapy ${ }^{[9]}$. Moreover, PTX may cause severe neurotoxicity by inhibiting neuronal microtubules, causing axonal degeneration, and leading to demyelination (especially peripheral neuropathy and ophthalmic neuropathy). Furthermore, hematological toxicity (myelosuppression), hair loss (even total alopecia), nausea, diarrhea, stomatitis, increase in liver enzymes, orthostatic hypotension, confusion, and encephalopathy are considered as common side effects. Although current treatment options may create remission in cancer patients, the above-mentioned side effects present a major problem and limit the ability of physicians to use available treatment options. There are different options to overcome this problem, including combinatory therapy ${ }^{[10,11]}$. That is why the combined treatment with some sensitizing agents is desirable to overcome these side effects and the resistance to PTX and increase the antitumor effects ${ }^{[2]}$.

Cancer cells, which are often genetically unstable and rapidly proliferative, tend to be more dependent on the proteasome than normal cells for the need of removing aberrant intracellular proteins. Thus, inhibition of the

*Address for correspondence E-mail: dr.terzi@hotmail.com November-December 2019
26S proteasome become an attractive approach for anticancer therapy ${ }^{[13]}$. The proteasome is a protease complex having a major role in cellular activities, including the processing of misfolded, unassembled, or damaged intracellular proteins. The accumulation of substrate proteins and, finally, cell death, can be observed by proteasome inhibition ${ }^{[12]}$. Carfilzomib (CFZ) is a second-generation proteasome inhibitor as a single agent in patients with relapsed/refractory multiple myeloma ${ }^{[14]}$. This agent has also been studied in various solid tumors and clinical trials, yet they have not clearly shown antitumor activity in solid tumors. At present, the effect of CFZ on breast cancer cell proliferation remains uncertainty ${ }^{[12]}$.

To date to our knowledge, no published studies have reported the possible effect of irreversible proteasome inhibitor CFZ and PTX combination against breast cancer. Therefore, the present study was conducted to investigate the possible synergistic effect of PTX plus CFZ combination on MDA-MB-231 breast cancer cells.

Human breast cancer cell line MDA-MB-231 (HTB-26) was acquired from American Type Culture Collection (ATCC, Manassas, VA, USA). PTX and CFZ were purchased from Sigma-Aldrich (St. Louis, MO, USA). The cells were maintained in Dulbecco's modified Eagle medium (DMEM; Lonza, Walkersville, MD, USA), which was supplemented with $10 \%(\mathrm{v} / \mathrm{v})$ heatinactivated fetal bovine serum (FBS; Sigma-Aldrich St. Louis, MO, USA) and $1 \%$ penicillin/streptomycin (Gibco Thermo Fisher Scientific). The cells were cultured in a $25 \mathrm{~cm}^{2}$ cell culture flask and incubated at $37^{\circ}$ in a $5 \% \mathrm{CO}_{2}$ humidified atmosphere until they reached approximately an 80-90\% confluence.

The cell viability was assessed using the XTT (2,3-bis(2-methoxy-4-nitro-5-sulfophenyl)-2H-tetrazolium5-carboxanilide) assay (Roche Diagnostic, Germany). PTX and CFZ were dissolved in dimethyl sulfoxide, the final concentration of which did not exceed $0.1 \%$ and diluted in DMEM before treatment. Cells were seeded in 96 -well plates at the density of $1 \times 10^{4}$ cells per well

This is an open access article distributed under the terms of the Creative Commons Attribution-NonCommercial-ShareAlike 3.0 License, which allows others to remix, tweak, and build upon the work non-commercially, as long as the author is credited and the new creations are licensed under the identical terms

Accepted 03 October 2019

Revised 04 July 2019

Received 19 March 2019 Indian J Pharm Sci 2019;81(6):1146-1153 
in $100 \mu \mathrm{l}$ of DMEM culture media and were allowed to adhere overnight. The next day, the cells were treated with increasing concentrations $(0.01,0.1,1,10$, $50 \mu \mathrm{M})$ of individual drugs alone or in combination $(0.005+0.005, \quad 0.05+0.05,0.5+0.5,5+5$, and $25+$ $25 \mu \mathrm{M})$ and the plates were then incubated for $48 \mathrm{~h}$. At the end of the incubation period, $50 \mu \mathrm{l}$ XTT labeling mixture was added to each well for determination of living cells, and then the plates were incubated at $37^{\circ}$ for another $4 \mathrm{~h}$. After mixing, the absorbance of each well was measured using a microplate reader (Thermo, Germany) at $450 \mathrm{~nm}$ against the control. All experiments were conducted in three independent experiments, and the cell viability was expressed in \% related to control (100\% of viability).

Combination index (CI) values were calculated according to the method of Chou and Talalay using the Compusyn software. $\mathrm{CI}<1, \mathrm{CI}=1$ and $\mathrm{CI}>1$ indicate synergistic effect, additive effect, and antagonistic effect, respectively ${ }^{[15]}$.

For apoptosis detection, the Muse Annexin V/dead cell assay (Merck Millipore) was utilized. MDA-MB-231 cells were seeded in a 6-well plate at a density of $1 \times 10^{6}$ cell per well and incubated overnight for attachment. After incubation, the cells were treated with $0.01,0.1$ or $1 \mu \mathrm{M}$ PTX alone, $0.005 \mu \mathrm{M} \mathrm{CFZ}$ alone or their combinations PTX $+\mathrm{CFZ}(0.005+0.05$ and $0.005+$ $0.005 \mu \mathrm{M}$ ) for $48 \mathrm{~h}$. After treatment, the cells were collected, diluted with PBS containing $1 \%$ FBS, and maintained with Muse ${ }^{\mathrm{TM}}$ Annexin V and Dead Cell reagent for $20 \mathrm{~min}$ at room temperature in the dark. Muse $^{\mathrm{TM}}$ Cell Analyzer analyzed the events for a living, dead, early and late apoptotic cells.

Cell cycle arrest was evaluated by Muse Cell Cycle assay kit (Merck Millipore, Germany) according to the user's guide. Initially, the cells were seeded and incubated overnight for attachment. Next day, the cells were treated with $0.1 \mu \mathrm{M}$ PTX alone, $0.005 \mu \mathrm{M}$ $\mathrm{CFZ}$ alone or 0.1 plus $0.005 \mu \mathrm{M}$ their combinations and incubated for $48 \mathrm{~h}$ at $37^{\circ}$. After completion of the incubation, the cells were collected by centrifugation $(300 \times \mathrm{g}, 5 \mathrm{~min})$, washed with cold PBS and fixed in chilled $70 \%(\mathrm{v} / \mathrm{v})$ ethanol for at least $3 \mathrm{~h}$ at $-20^{\circ}$ before staining. Next, fixed cells were washed once with PBS and incubated with $200 \mu$ of assay solution for $30 \mathrm{~min}$ in the dark at room temperature according to the manufacturer's protocol. After staining, the percentage of the cells at different stages of the cell cycle (G0/G1, $\mathrm{S}$, and G2/M phases) was analyzed by using the Muse Cell Analyzer (Merck Millipore).
The statistical significance for the assays was determined using GraphPad Prism 7 (GraphPad Software, Inc.). Data obtained from the cell viability experiments were expressed as the mean \pm standard deviation, and oneway ANOVA test was applied for multiple comparisons. The value of $p$ less than or equal to 0.01 was considered statistically significant.

In the present study, the first aim was to evaluate the inhibitory effects of PTX and CFZ on MDA-MB-231 breast cancer cells by applying the drugs in monotherapy and in combination for $48 \mathrm{~h}$. Initially, dose-response experiments were performed with each drug alone. The cells were treated with an increased concentration of $0.01,0.1,1,10$ or $50 \mu \mathrm{M}$ of PTX alone and $0.01,0.1,1$, 10 or $50 \mu \mathrm{M}$ of CFZ alone and calculated $\mathrm{IC}_{50}$ values. As seen in fig. 1, PTX and CFZ alone significantly inhibited cell growth at $0.01 \mu \mathrm{M}$ and higher concentrations for $48 \mathrm{~h}$ in a dose-dependent manner. The $\mathrm{IC}_{50}$ values of PTX and CFZ were calculated as $5.02 \mu \mathrm{M}$ and $0.079 \mu \mathrm{M}$, respectively.

The second aim was to verify whether PTX and CFZ have synergistic effects on MDA-MB-231 cells. In order to determine the synergistic effect between PTX and CFZ, the cells were treated with $(0.005,0.05,0.5$, 5 or $25 \mu \mathrm{M})$ PTX plus $(0.005,0.05,0.5,5$ or $25 \mu \mathrm{M})$ $\mathrm{CFZ}$ combination for $48 \mathrm{~h}$. The results demonstrated that combination treatment significantly inhibited breast cancer cells growth when compared to PTX or CFZ alone (fig. 1).

The CI values were calculated by the Compusyn software and all combinations of PTX with CFZ gave a CI value less than 1 , indicating a synergistic effect of the 2 drugs on breast cancer cells (fig. 2). These observations clearly suggested that CFZ could sensitize the cytotoxicity of PTX on the MDA-MB-231 breast cancer cells. Furthermore, we would like it if other

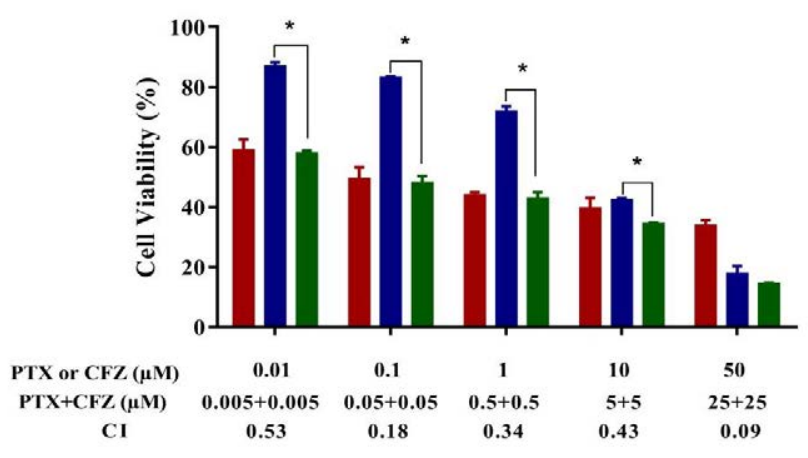

Fig. 1: Antiproliferative activity of PTX, CFZ, and their combination on MDA-MB-231 cells for $48 \mathrm{~h}$

The differences are identified as *from PTX, $p<0.01$, ( $\square$ ) CFZ, (घ) PTX, ( ) PTX+CFZ

November-December 2019 
researchers confirm it using different methods such as molecular docking.

Before apoptosis experiments, the MDA-MB-231 cells were treated with $0.01,0.1$ or $1 \mu \mathrm{M}$ of PTX alone, $0.005 \mu \mathrm{M}$ of CFZ alone or in combination with CFZ at a constant concentration $(0.005 \mu \mathrm{M})$ for $48 \mathrm{~h}$. Afterward, Annexin $\mathrm{V}$ binding assay was performed to evaluate the effects of the drugs on the apoptosis of MDAMB-231 cells. In this assay, 4 populations of cells can be distinguished, namely non-apoptotic cells, Annexin V (-) and 7-AAD (-), early apoptotic cells, Annexin $\mathrm{V}(+)$ and 7-AAD (-), late-stage apoptotic cells, Annexin $\mathrm{V}(+)$ and 7-AAD $(+)$ and necrotic cells, Annexin $\mathrm{V}(-)$ and 7-AAD $(+)$. As seen in fig. 3, when compared to PTX or CFZ alone, combinations of 0.005 and $0.005 \mu \mathrm{M}$ PTX + CFZ or 0.05 and $0.005 \mu \mathrm{M}$ PTX + CFZ caused a significantly higher apoptotic effect $(\mathrm{p}<0.01)$.

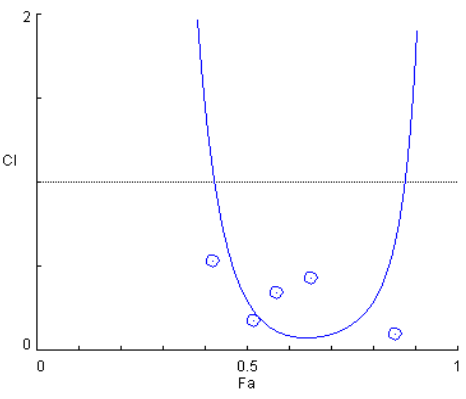

Fig. 2: Combination index (CI) plot

The synergistic effect of PTX in combination with CFZ on the growth of MDA-MB-231 cell line. Combination index (CI) values were calculated at the drug concentration of PTX $(0.005 \mu \mathrm{M})$ plus CFZ $(0.005 \mu \mathrm{M})$, PTX (0.05 $\mu \mathrm{M})$ plus CFZ $(0.05 \mu \mathrm{M})$, PTX $(0.5 \mu \mathrm{M})$ plus CFZ $(0.5 \mu \mathrm{M})$, PTX $(5 \mu \mathrm{M})$ plus CFZ $(5 \mu \mathrm{M})$, and PTX $(25 \mu \mathrm{M})$ plus CFZ $(25 \mu \mathrm{M})$ using the ChouTalalay method. Drug synergy, addition, and antagonism are determined by CI values less than 1.0, equal to $\mathbf{1 . 0}$, or greater than 1.0, respectively

(A)

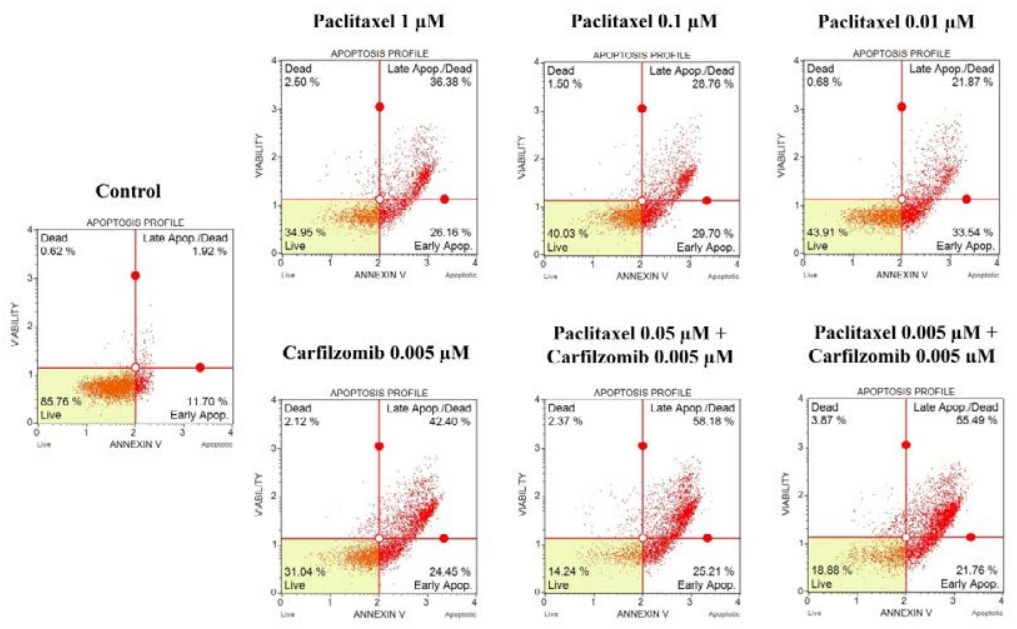

(B)

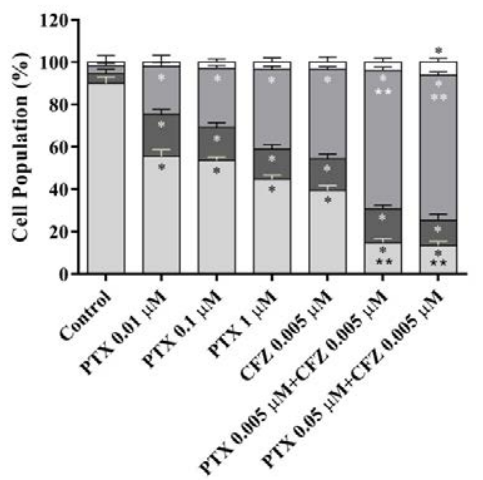

Fig. 3: (A) apoptotic effects of CFZ, PTX and their combination on MDA-MB-231 cells, (B) percentage of non-apoptotic cells, early apoptotic cells, late-stage apoptotic cells, and necrotic cells

A: cells were treated at the indicated concentrations of CFZ, PTX, or their combination for $48 \mathrm{~h}$. After the incubation time, the cells were stained using the Muse Annexin V and Dead cell reagent, and then \% gated values were evaluated by using Muse Cell Analyzer (Merck Millipore). B: all experiments were carried out triplicate, and similar results were obtained. Statistically, significant differences are $* \mathbf{p}<0.01$ from values compared to the control group, ${ }^{* *} \mathbf{p}<0.01$ from values compared to the PTX $+\mathrm{CFZ}$ combination and PTX alone; $(\square)$ debris, ( $\square$ ) late apoptotic/dead, ( $\square$ ) early apoptotic, ( $\square$ ) live 
The total apoptotic cell population percentages were determined as 44.58, 46.4, 55.35, and $60.63 \%$, respectively in MDA-MB-231 cells treated with PTX $0.01,0.1,1 \mu \mathrm{M}$ and CFZ $0.005 \mu \mathrm{M}$ alone. Furthermore, when both drugs are combined, total apoptotic rates were increased to 85.21 and $86.57 \%$, respectively in PTX plus CFZ $(0.005+0.005 \mu \mathrm{M})$ and PTX plus CFZ $(0.05+0.005 \mu \mathrm{M})$ treated groups $(\mathrm{p}<0.01)$. Besides, due to the apoptotic effects of $0.5+0.005 \mu \mathrm{M}$ PTX $+\mathrm{CFZ}$ combination on MDA-MB-231 cells was very strong, the apoptosis assay of this combination could not be performed.

Previous experiments revealed that PTX $+\mathrm{CFZ}$ combination inhibited cell proliferation and induced apoptosis significantly when compared to PTX and CFZ alone. In the next step, the effect of the compounds on cell cycle distribution was determined by using the cell cycle kit to investigate whether or not the PTX + CFZ combination induced growth inhibition of cells was mediated via alterations in the cell cycle. The results showed that $48 \mathrm{~h}$ later, the $\mathrm{G} 2 / \mathrm{M}$ phase population of control cells was $23.7 \pm 1.5 \%$ and the percentages significantly increased to $57.6 \pm 1.9,30.3 \pm 1.1$ and $83.8 \pm 1.4 \%$ for PTX, CFZ, and their combinations, respectively (fig. 4). The cell cycle results revealed that the PTX + CFZ combination significantly increases of cell population at $\mathrm{G} 2 / \mathrm{M}$ phase when compared to the control or PTX and CFZ alone group.

It is very well known in the literature and clinical practice that PTX has a broad spectrum and an effective antitumor effect on lung cancer, breast cancer, ovarian cancer, melanoma, cervical cancer, and many other tumors ${ }^{[16]}$. Although PTX is a very potent and effective anticancer agent, it also has strong side effects including hematological toxicity (myelosuppression), hair loss (even total alopecia), nausea, diarrhea, stomatitis, increase in liver enzymes, orthostatic hypotension, confusion, and encephalopathy. PTX may also cause severe neurotoxicity by inhibiting neuronal microtubules, causing axonal degeneration, and leading to demyelinization (especially peripheral neuropathy and ophthalmic neuropathy). Moreover, single administration may create drug resistance which may be related to poor prognosis ${ }^{[17,18]}$. These

(A)
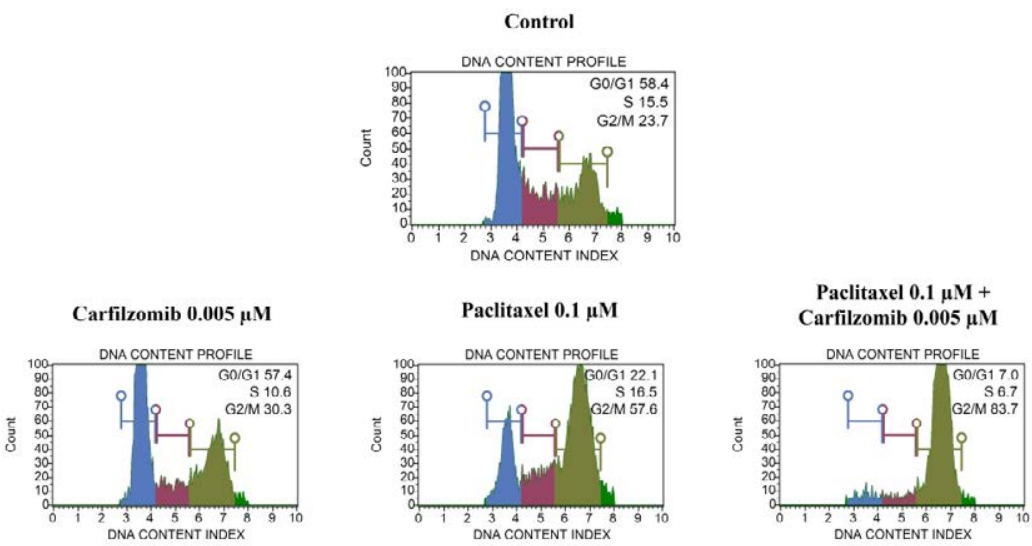

(B)

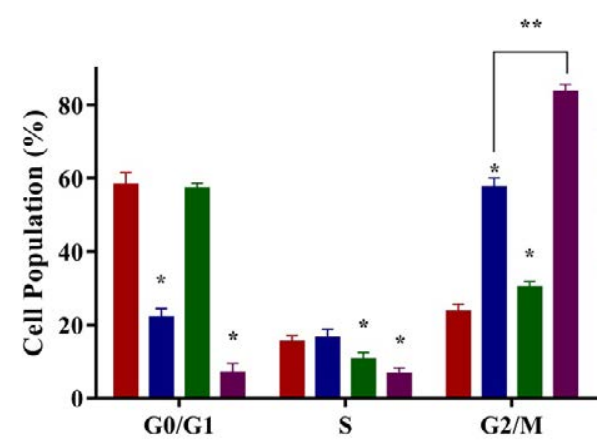

Fig. 4: Effects of PTX, CFZ alone and in combination on cell cycle distribution and \% cell cycle phases in MDA-MB-231 cells (A) effects of PTX, CFZ, and their combination on cell cycle distribution in MDA-MB-231 cells for $48 \mathrm{~h}$, (B) histogram display of

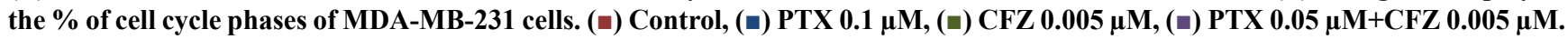
Results are expressed as mean \pm SD. The differences are given as $* \mathbf{p}<0.01$ compared to the control group and $* * p<0.01$ compared to the PTX+CFZ combination and PTX alone 
issues may limit the clinical use of PTX and highlight a requirement for new approaches in breast cancer treatment, such as combination therapy ${ }^{[19]}$. Therefore, novel therapeutic strategies especially discover new combinations for breast cancer treatment are urgently needed to overcome these side effects.

Bortezomib is a first-generation proteasome inhibitor, used for the treatment of multiple myeloma and relapsed or refractory mantle cell lymphoma ${ }^{[20]}$. Secondgeneration proteasome inhibitor CFZ is the second FDA-approved proteasome inhibitor for the treatment of recurrent multiple myeloma. There are many studies in the literature in which CFZ has been used to treat a different kind of solid tumors ${ }^{[21,22]}$, yet they have not shown consistent antitumor activity in solid tumors ${ }^{[12]}$. Furthermore, combinations of proteasome inhibitors and other chemotherapeutic agents have been examined for use in cancer treatment. Development of proteasome inhibitors with distinct substrate selectivity, increased bioavailability, and lower toxicity may open the passage to extensive usage in solid tumors ${ }^{[23]}$.

To date, several studies have been conducted combining CFZ with various antineoplastic drugs on various solid tumors ${ }^{[24,25]}$. However, even today, the effect of $\mathrm{PTX}+\mathrm{CFZ}$ on breast cancer cell proliferation remains unclear. Here, antitumor, apoptotic, and cell cycle arrest effects of PTX + CFZ on the breast cancer cells were examined. According to the results of the present study, administration of both PTX and CFZ alone created a concentration-dependent anticancer effect on breast cancer cells. Additionally, it was found that PTX $+\mathrm{CFZ}$ combination had high cytotoxic activity against the MDA-MB-231 cells compared to PTX or CFZ alone (CI values for all combinations of CFZ and PTX were far lower than 1.0). This synergistic effect was also detected by the apoptotic effects of the PTX and CFZ. The apoptotic effects of PTX alone, CFZ alone or their combination were evaluated on MDA-MB-231 cells, and these results exhibited that PTX $+\mathrm{CFZ}$ combination increases the apoptotic response when compared to PTX or CFZ alone. These cell cycle experiments also suggested that $\mathrm{PTX}+\mathrm{CFZ}$ combination results in a significant increase of cell population at the $\mathrm{G} 2 / \mathrm{M}$ phase compared to control and PTX or CFZ alone. In the previous studies, it was shown that PTX or CFZ created a cell cycle arrest at the G2/M phase ${ }^{[26,27]}$. Similarly, in the present study, it was determined that both CFZ and PTX created a cell cycle arrest at G2/M phase. Hence, the combination of CFZ and PTX doubled the number of cells arrested at $\mathrm{G} 2 / \mathrm{M}$ phase, which might explain the increase in anticancer effect via combination.

Many studies are indicating that PTX has strong apoptotic effects on various cancer cell types ${ }^{[28,29]}$. In a recent study, it has been reported that CFZ enhanced the cytotoxic effect of doxorubicin and sensitizes neuroblastoma cells to doxorubicin. In the same study, it has been shown that CFZ significantly induces apoptosis in neuroblastoma cells ${ }^{[24]}$. In a similar fashion, Shi et al. ${ }^{[25]}$ demonstrated that CFZ intensifies the cytotoxic effect of doxorubicin and inhibit cell proliferation synergistically on breast cancer cells. In another study, Jiang and colleagues ${ }^{[30]}$ investigated whether sorafenib and CFZ act synergistically to inhibit the proliferation of hepatocellular carcinoma (HCC) cells. Their results have displayed that a combination of sorafenib and CFZ has synergistic antitumor activities against HCC cells when compared to sorafenib or CFZ alone. Ashley et $a l .^{[31]}$ have indicated that the combination of CFZ plus doxorubicin-loaded liposomal nanoparticles synergistically inhibit the proliferation of multiple myeloma cells. Furthermore, in the pertinent literature, there are several clinical data rather than in vitro studies regarding the synergistic effect of CFZ and various antineoplastic drugs ${ }^{[32]}$.

In accordance with the literature, this study suggests that PTX had a potent anticancer and apoptotic effects on breast cancer cells. Moreover, the present study shows the synergistic activity of PTX when combined with CFZ. Increased side effect related to the use of high dose PTX is one of the most challenging scenarios in breast cancer chemotherapy. The dosage needs to be reduced when the side effects occur, and sometimes it is required to be discontinued if the side effects become intolerable.

In this study, significant anticancer activity was observed when PTX and CFZ were administrated in combination with the reduced doses. Since the combination of PTX and CFX showed promising anticancer effect at a considerably low dose, this may decrease the incidence of side effects associated with the drugs. As a result, this combination could potentially show higher anticancer efficacy with less toxicity.

In summary, the results of the present study indicated that CFZ could significantly increase the efficacy of PTX's anticancer activity in human breast cancer cells in vitro. According to experimental results, it was clearly shown that the combination of PTX $+\mathrm{CFZ}$ had high cytotoxic activity against the MDA-MB-231 compared to PTX or 
CFZ alone. In light of this evidence, it is recommended that CFZ may be used along with PTX in metastatic breast cancer patients to increase the anticancer effect and reduce potential toxicity-related side effects. However, this synergistic effect could be supported by different methods, such as molecular docking. Besides, since this is an in vitro study indicating the effect and mechanism of action of the combination approach, more clinical studies are needed to prove the value of this combination in clinical practice.

\section{Conflicts of interest:}

The authors confirm that this article content has no conflict of interest.

\section{Financial support and sponsorship:}

Nil.

\section{REFERENCES}

1. Siegel R, Naishadham D, Jemal A. Cancer statistics, 2013. CA Cancer J Clin 2013;63:11-30.

2. Mohan A, Ponnusankar S. Newer therapies for the treatment of metastatic breast cancer: a clinical update. Indian J Pharm Sci 2013;75:251-61.

3. Li Y, Brasky TM, Nie J, Ambrosone CB, McCann SE, Shields PG, et al. Use of nonsteroidal anti-inflammatory drugs and survival following breast cancer diagnosis. Cancer Epidemiol Biomarkers Prev 2012;21:239-42.

4. Wei XQ, Li X, Xin XJ, Tong ZS, Zhang S. Clinical features and survival analysis of very young $(a g e<35)$ breast cancer patients. Asian Pac J Cancer Prev 2013;14:5949-52.

5. Sung M, Giannakakou P. BRCA1 regulates microtubule dynamics and taxane-induced apoptotic cell signaling. Oncogene 2014;33:1418-28.

6. Carbognin L, Sperduti I, Nortilli R, Brunelli M, Vicentini $\mathrm{C}$, Pellini $\mathrm{F}$, et al. Balancing activity and tolerability of neoadjuvant paclitaxel- and docetaxel-based chemotherapy for HER2-positive early stage breast cancer: sensitivity analysis of randomized trials. Cancer Treat Rev 2015;41:262-70.

7. Tang S, Yin Q, Su J, Sun H, Meng Q, Chen Y, et al. Inhibition of metastasis and growth of breast cancer by $\mathrm{pH}$-sensitive poly ( $\beta$-amino ester) nanoparticles co-delivering two siRNA and paclitaxel. Biomaterials 2015;48:1-15.

8. Zhou N, Liu C, Hou H, Zhang C, Liu D, Wang G, et al. Response to apatinib in chemotherapy-failed advanced spindle cell breast carcinoma. Oncotarget 2016;7:72373-79.

9. Valastyan S, Weinberg RA. Tumor metastasis: molecular insights and evolving paradigms. Cell 2011;147:275-92.

10. Rivera E, Cianfrocca M. Overview of neuropathy associated with taxanes for the treatment of metastatic breast cancer. Cancer Chemother Pharmacol 2015;75:659-70.

11. Ojima I, Lichtenthal B, Lee S, Wang C, Wang X. Taxane anticancer agents: a patent perspective. Expert Opin Ther Pat 2016;26:1-20.

12. Khan ML, Stewart AK. Carfilzomib: a novel second-generation proteasome inhibitor. Future Oncol 2011;7:607-12.

13. Chang HY, Huang TC, Chen NN, Huang HC, Juan HF. Combination therapy targeting ectopic ATP synthase and $26 \mathrm{~S}$ proteasome induce ER stress in breast cancer cells. Cell Death Dis 2014;27:1540.

14. Engelhardt M, Szymaniak-Vits M, Ajayi S, Dold SM, Müller SJ, Scheubeck S, et al. Carfilzomib. Recent Results. Cancer Res 2018;212:265-83.

15. Chou TC, Talalay P. Quantitative analysis of dose-effect relationships: The combined effects of multiple drugs or enzyme inhibitors. Adv Enzyme Regulat 1984;22:27-55.

16. Ye J, Dong W, Yang Y, Hao H, Liao H, Wang B, et al. Vitamin E-rich Nanoemulsion Enhances the Antitumor Efficacy of Low-Dose Paclitaxel by Driving Th1 Immune Response. Pharm Res 2017;34:1244-54.

17. Frederiks CN, Lam SW, Guchelaar HJ, Boven E. Genetic polymorphisms and paclitaxel- or docetaxel-induced toxicities. A systematic review. Cancer Treat Rev 2015;41:935-50.

18. Alves RC, Fernandes RP, Eloy JO, Salgado HRN, Chorilli M. Characteristics, Properties and Analytical Methods of Paclitaxel: A Review. Crit Rev Anal Chem 2018;48:110-8.

19. Jabir RS, Naidu R, Annuar MA, Ho GF, Munisamy M, Stanslas J. Pharmacogenetics of taxanes: impact of gene polymorphisms of drug transporters on pharmacokinetics and toxicity. Pharmacogenomics 2012;13:1979-88.

20. Roccaro AM, Vacca A, Ribatti D. Bortezomib in the treatment of cancer. Recent Pat Anticancer Drug Discov 2006;1:397-403.

21. Kuhn DJ, Orlowski RZ, Bjorklund CC. Second generation proteasome inhibitors: carfilzomib and immunoproteasomespecific inhibitors (IPSIs). Curr Cancer Drug Targets 2011;11:285-95.

22. Yang H, Zonder JA, Dou QP. Clinical development of novel proteasome inhibitors for cancer treatment. Expert Opin Investig Drugs 2009;18:957-71.

23. Manasanch EE, Orlowski RZ. Proteasome inhibitors in cancer therapy. Nat Rev Clin Oncol 2017;14:417-33.

24. Guan S, Zhao Y, Lu J, Yu Y, Sun W, Mao X, et al. Secondgeneration proteasome inhibitor carfilzomib sensitizes neuroblastoma cells to doxorubicin-induced apoptosis. Oncotarget 2016;7:75914-25.

25. Shi Y, Yu Y, Wang Z, Wang H, Bieerkehazhi S, Zhao Y, et al. Second-generation proteasome inhibitor carfilzomib enhances doxorubicin-induced cytotoxicity and apoptosis in breast cancer cells. Oncotarget 2016;7:73697-710.

26. Huang MB, Gonzalez RR, Lillard J, Bond VC. Secretion modification region-derived peptide blocks exosome release and mediates cell cycle arrest in breast cancer cells. Oncotarget 2017;8:11302-15.

27. Gu JJ, Hernandez-Ilizaliturri FJ, Kaufman GP, Czuczman NM, Mavis C, Skitzki JJ, et al. The novel proteasome inhibitor carfilzomib induces cell cycle arrest, apoptosis and potentiates the anti-tumour activity of chemotherapy in rituximab-resistant lymphoma. Br J Haematol 2013;162:657-69.

28. Rege MD, Ghadi R, Katiyar SS, Kushwah V, Jain S. Exploring an interesting dual functionality of anacardic acid for efficient paclitaxel delivery in breast cancer therapy. Nanomedicine 2019;14:57-75.

29. Wang C, Su K, Zhang Y, Zhang W, Chu D, Zhao Q, et al. MicroRNA-365 targets multiple oncogenes to inhibit proliferation, invasion, and self-renewal of aggressive endometrial cancer cells. Cancer Manag Res 2018;10:5171-85.

30. Jiang C, Xu R, Li XX, Zhou YF, Xu XY, Yang Y, et al. Sorafenib and Carfilzomib Synergistically Inhibit the Proliferation, Survival, and Metastasis of Hepatocellular Carcinoma. Mol Cancer Ther 2018;7:2610-21. 
31. Ashley JD, Quinlan CJ, Schroeder VA, Suckow MA, Pizzuti VJ, Kiziltepe T, et al. Dual Carfilzomib and DoxorubicinLoaded Liposomal Nanoparticles for Synergistic Efficacy in Multiple Myeloma. Mol Cancer Ther 2016;15:1452-9.

32. Arnold SM, Chansky K, Leggas M, Thompson MA, Villano
$\mathrm{JL}$, Hamm $\mathrm{J}$, et al. Phase $1 \mathrm{~b}$ trial of proteasome inhibitor carfilzomib with irinotecan in lung cancer and other irinotecansensitive malignancies that have progressed on prior therapy (Onyx IST reference number: CAR-IST-553). Invest New Drugs 2017;35:608-15. 